\title{
Response of Pre and Post-Harvest Chemical Applications on Storage Life, Quality and Cost Economics of Mango cv. Dashehari in Tarai Region of Uttarakhand, India
}

\author{
Pant*, Pooja and C.P. Singh
}

Department of Horticulture, G.B. Pant University of Agriculture \& Technology, Pantnagar, US Nagar, Uttarakhand-263145, India

*Corresponding author

\section{A B S T R A C T}

\begin{tabular}{|l|}
\hline Ke y w or d s \\
$\begin{array}{l}\text { Mango, } \mathrm{CaCl}_{2}, \mathrm{GA}_{3}, \\
\text { Dashehari, Quality }\end{array}$ \\
\hline Article Info \\
\hline $\begin{array}{l}\text { Accepted: } \\
22 \text { October } 2018 \\
\text { Available Online: } \\
10 \text { November } 2018\end{array}$ \\
\hline
\end{tabular}

\section{Introduction}

Mango is well established for international trade due to its superb quality. The export market for mango has become highly lucrative. Domestic and international trade of fresh mango has been limited by its highly perishable nature and susceptibility to postharvest diseases and physical injuries. Postharvest losses have been estimated in developed countries from $5-25 \%$, while in developing countries it is near to $20-50 \%$ (Cisneros-Zevallos, 2003). The losses after harvesting of fruits are more due to mismanagement of produce and unfavourable physiological, biological and environmental factors.
The present investigation was undertaken on mango cv. Dashehari with the objective to find out suitable treatments for better storage life, quality and net profit of mango fruits. he experiment was laid out in factorial completely randomized design comprising of 8 post-harvest treatments. The pre harvest application of $\mathrm{GA}_{3} @ 15 \mathrm{ppm}+\mathrm{CaCl}_{2}$ C respectively) and maximum shelf life (13.25 days), total sugars (18.76\% and $17.76 \%$, respectively), ascorbic acid $\left(45.78 \mathrm{mg} 100 \mathrm{~g}^{-1}\right.$ and $46.47 \mathrm{mg} 100 \mathrm{~g}^{-1}$, respectively) and $\beta-$

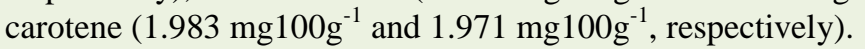


2013 on mango cv. Dashehari. The climate of the area is humid subtropical with dry hot summers $\left(32^{0} \mathrm{C}-45^{\circ} \mathrm{C}\right)$ and cool winters $\left(0^{0} \mathrm{C}\right.$ $\left.9^{0} \mathrm{C}\right)$. Frost can be expected from last week of December to middle of February. Rainy season is usually from 2nd week of June to 2nd week of September with heavy rainfall $(1400 \mathrm{~mm})$. The experiment was laid out in CRD factorial comprising of 8 pre and 10 post-harvest treatments (mentioned in tables) and each treatment replicated thrice. The preharvest foliar spray of chemicals on 25 years old trees of mango cv. Dashehari was done 20 days before harvesting of fruits, while, the post-harvest treatment in form of dipping in different chemicals dipping was done after harvesting and kept in ventilated CFB boxes and stored at ambient temperature $\left(30-36^{\circ} \mathrm{C}\right)$ for further studies. Fruit setting was started in Februrary- March. And fully matured fruits were picked in last week of June. During maturation phase (from 18 to 25 standard week) maximum temperature varied from 38.3 to $34.1^{\circ} \mathrm{C}$ and 36.1 to $41.1^{\circ} \mathrm{C}$, minimum $22.0-$ $25.6^{\circ} \mathrm{C}$ and 18.6 to $27.8^{\circ} \mathrm{C}$, relative humidity varied from $63-82 \%$ (morning) to $31-61 \%$ (evening) and $47-66 \%$ (morning) to $18-41 \%$ (evening), sunshine hours from 6.6 to $9.5 \mathrm{hrs}$ and 3.3 to $10.6 \mathrm{hrs}$ during $1^{\text {st }}$ and $2^{\text {nd }}$ season, respectively. The total rainfall during this period was $156.6 \mathrm{~mm}$ and $21.2 \mathrm{~mm}$.

Treatment wise, the mango fruits of both the experiments were stored in a clean, hygienic and well ventilated room at ambient condition (temperature $31 \pm 1{ }^{\circ} \mathrm{C}$ and humidity $60 \pm 5 \%$ ). Observations on storage life (D), total sugars (\%), ascorbic acid $\left(\mathrm{mg} 100 \mathrm{~g}^{-1}\right)$ and $\beta-$ carotene $\left(\mathrm{mg}_{\left.100 \mathrm{~g}^{-1}\right)}\right.$ were recorded. The total sugars, ascorbic acid and $\beta$ - carotene was determined as per AOAC (1990), while, economics was calculated on the basis of market rate prevailed at that period. The data was statistically analysed to know the significance among the mean values of the treatments.

\section{Results and Discussion}

The data on storage life and total sugars are summarized in Table 1. The pre harvest spray and post-harvest dipping of chemicals enhanced storage life of fruits under all the treatments over control. The storage life of fruits treated with chemicals could be extended up to 13 days over 7 days of untreated ones (control). The longest storage life (13.25 days) was observed with the pre harvest application of $\mathrm{GA}_{3} @ 15$ ppm + $\mathrm{CaCl}_{24} @ 2.0 \%$ and post-harvest dipping with $\mathrm{GA}_{3} @ 400$ ppm + $\mathrm{CaCl}_{2} @ 6.0 \%\left(\mathrm{~T}_{3}\right)$ and hot water+ $\mathrm{CaCl}_{2} @ 6.0 \%\left(\mathrm{~T}_{9}\right)$.

The increase in storage life might be due to less weight loss, spoilage, shrivelling and enhanced fruit firmness, which provide improved fruit colour, better appearance, glossiness and enhanced market acceptability (Choudhury et al., 2003).

During the storage durations, the total sugar contents of the fruits increased gradually with advancement of storage period and reached to the maximum levels $(18.76 \%$ and $17.76 \%$, respectively) on $9^{\text {th }}$ day of storage with the pre harvest spray of $\mathrm{GA}_{3} @ 15 \mathrm{ppm}+\mathrm{CaCl}_{2} @$ $2.0 \%\left(\mathrm{~T}_{3}\right)(0.291 \%)$ and post-harvest dipping with hot water $+\mathrm{CaCl}_{2} @ 6.0 \%\left(\mathrm{~T}_{9}\right)(0.310 \%)$. The increase in total sugar may be due to breakdown of complex polymers in to simple substances by hydrolytic enzymes (Mahajan et al., 2004; Wahdan et al., 2011).

The data on ascorbic acid $\left(\mathrm{mg} 100 \mathrm{~g}^{-1}\right)$ and $\beta-$ carotene $(\mathrm{mg} / 100 \mathrm{~g})$ are summarized in Table 2. The pre harvest spray of $\mathrm{GA}_{3} @ 15 \mathrm{ppm}+$ $\mathrm{CaCl}_{2} @ 2.0 \%\left(\mathrm{~T}_{3}\right)$ and post-harvest dipping with $\mathrm{GA}_{3} @ 400$ ppm + $\mathrm{CaCl}_{2} @ 6.0 \%\left(\mathrm{~T}_{3}\right)$ registered a very low and gradual decline in ascorbic acid content with advancement of storage period and was recorded 45.78 $\mathrm{mg} 100 \mathrm{~g}^{-1}$ and $46.47 \mathrm{mg}_{100 \mathrm{~g}^{-1}}$, respectively on the day of harvest. 
Table.1 Effect of pre and post-harvest treatments on storage life and total sugar percent of mango cv. Dashehari during storage (Pooled analysis)

\begin{tabular}{|c|c|c|c|c|c|c|c|c|}
\hline \multirow[t]{4}{*}{ Treatments (T) } & \multirow{4}{*}{$\begin{array}{l}\text { Storage } \\
\text { Life(D) }\end{array}$} & \multicolumn{7}{|c|}{ Total Sugars $(\%)$} \\
\hline & & \multicolumn{7}{|c|}{ Storage intervals in days (D) } \\
\hline & & $\mathbf{0}$ & 3 & 6 & 9 & 12 & 14 & Mean \\
\hline & & \multicolumn{7}{|c|}{ Preharvest } \\
\hline $\mathrm{T}_{1}: \mathrm{CaCl}_{2} @ \mathbf{2 \%}$ & 12.00 & 9.02 & 10.93 & 14.56 & 16.90 & 16.47 & 15.81 & 13.95 \\
\hline $\mathrm{T}_{2}: \mathrm{GA}_{3} @ 15 p p m$ & 11.17 & 9.10 & 11.13 & 14.78 & 17.12 & 16.66 & 16.04 & 14.14 \\
\hline $\mathrm{T}_{3}: \mathrm{GA}_{3} @ 15 \mathrm{ppm}+\mathrm{CaCl}_{2} @ 2.0 \%$ & 13.25 & 9.51 & 12.77 & 15.95 & 18.76 & 18.33 & 17.75 & 15.51 \\
\hline $\mathrm{T}_{4}$ :Bavistin @ $0.5 \%$ & 9.00 & 9.20 & 11.69 & 14.59 & 16.74 & 16.30 & 15.64 & 14.03 \\
\hline $\mathrm{T}_{5}$ :Bavistin@ $0.5 \%+\mathrm{CaCl}_{2} @ 2.0 \%$ & 10.08 & 9.65 & 13.02 & 15.75 & 17.81 & 17.38 & 16.75 & 15.06 \\
\hline $\mathrm{T}_{6}$ :Indoneem @ 500 ppm & 8.00 & 9.37 & 12.19 & 14.94 & 17.07 & 16.61 & 15.96 & 14.36 \\
\hline $\mathrm{T}_{7}$ :Indoneem @ 500 ppm + $\mathrm{CaCl}_{2} @ 2.0 \%$ & 8.00 & 9.53 & 12.69 & 15.69 & 17.68 & 16.72 & 16.05 & 14.73 \\
\hline $\mathrm{T}_{8}:$ Control & 7.00 & 8.78 & 13.88 & 17.22 & 14.99 & 13.61 & 12.50 & 13.50 \\
\hline Mean & - & 9.27 & 12.29 & 15.44 & 17.13 & 16.51 & 15.81 & 14.41 \\
\hline \multirow{2}{*}{ CD at $5 \%(\mathrm{~T} \times \mathrm{D})$} & 0.728 & & & & 0.185 & & & \\
\hline & & \multicolumn{7}{|c|}{ Post-harvest } \\
\hline $\mathrm{T}_{1}: \mathrm{CaCl}_{2} @ 6 \%$ & 12.17 & 9.37 & 11.51 & 13.91 & 16.71 & 16.08 & 15.12 & 13.78 \\
\hline$T_{2}: G_{3} @$ 400ppm & 11.17 & 9.38 & 11.65 & 14.33 & 16.45 & 15.84 & 14.83 & 13.74 \\
\hline $\mathrm{T}_{3}: \mathrm{GA}_{3} @ 400$ ppm + $\mathrm{CaCl}_{2} @ 6.0 \%$ & 13.25 & 9.58 & 11.33 & 13.54 & 17.35 & 16.85 & 16.00 & 14.11 \\
\hline $\mathrm{T}_{4}$ :Bavistin @ $0.1 \%$ & 11.17 & 9.67 & 11.39 & 13.69 & 16.99 & 16.43 & 15.56 & 13.95 \\
\hline $\mathrm{T}_{5}$ :Bavistin@0.1\% + $\mathrm{CaCl}_{2} @ 6.0 \%$ & 12.00 & 9.57 & 11.35 & 13.80 & 17.10 & 16.52 & 15.69 & 14.00 \\
\hline $\mathrm{T}_{6}:$ Indoneem @ 500 ppm & 8.50 & 9.53 & 11.91 & 15.29 & 15.92 & 15.21 & 14.03 & 13.65 \\
\hline $\mathrm{T}_{7}$ :Indoneem @ 500 ppm + $\mathrm{CaCl}_{2} @ 6.0 \%$ & 9.58 & 9.66 & 11.84 & 15.03 & 16.05 & 15.38 & 14.27 & 13.70 \\
\hline $\mathrm{T}_{8}$ :hot water & 13.00 & 9.40 & 11.17 & 13.33 & 17.58 & 17.08 & 16.27 & 14.14 \\
\hline $\mathrm{T}_{9}$ :hot water + $\mathrm{CaCl}_{2} @ 6.0 \%$ & 13.25 & 9.38 & 11.04 & 13.13 & 17.76 & 17.31 & 16.44 & 14.18 \\
\hline $\mathrm{T}_{10}:$ Control & 7.00 & 9.22 & 13.34 & 16.40 & 14.96 & 14.24 & 13.07 & 13.54 \\
\hline Mean & - & 9.47 & 11.65 & 14.24 & 16.69 & 16.09 & 15.13 & 13.88 \\
\hline CD at $5 \%(T \times D)$ & 0.678 & \multicolumn{7}{|c|}{0.087} \\
\hline
\end{tabular}


Table. 2 Effect of pre and post-harvest treatments on the Ascorbic acid and $\beta$-Carotene of mango cv.

Dashehari during storage (Pooled analysis)

\begin{tabular}{|c|c|c|c|c|c|c|c|c|c|c|c|c|c|c|}
\hline \multirow[t]{4}{*}{ Treatments (T) } & \multicolumn{7}{|c|}{ Ascorbic acid (mg100g $\left.\mathrm{g}^{-1}\right)$} & \multicolumn{7}{|c|}{$\beta$-Carotene (mg100g $\left.{ }^{-1}\right)$} \\
\hline & \multicolumn{7}{|c|}{ Storage intervals in days (D) } & \multicolumn{7}{|c|}{ Storage intervals in days (D) } \\
\hline & $\mathbf{0}$ & 3 & 6 & 9 & 12 & 14 & Mean & $\mathbf{0}$ & 3 & 6 & 9 & 12 & 14 & Mean \\
\hline & \multicolumn{14}{|c|}{ Pre harvest } \\
\hline $\mathrm{T}_{1}: \mathrm{CaCl}_{2} @ 2 \%$ & 43.85 & 38.64 & 31.47 & 27.09 & 22.81 & 20.31 & 30.69 & 0.294 & 0.875 & 1.562 & 1.947 & 1.928 & 1.904 & 1.418 \\
\hline $\mathrm{T}_{2}: \mathrm{GA}_{3} @$ 15ppm & 43.99 & 38.84 & 31.75 & 27.36 & 23.67 & 21.02 & 31.10 & 0.298 & 0.885 & 1.583 & 1.957 & 1.936 & 1.909 & 1.428 \\
\hline $\mathrm{T}_{3}: \mathrm{GA}_{3} @ 15 \mathrm{ppm}+\mathrm{CaCl}_{2} @ 2.0 \%$ & 45.78 & 40.55 & 35.00 & 30.47 & 26.05 & 23.51 & 33.56 & 0.293 & 0.918 & 1.641 & 1.983 & 1.967 & 1.948 & 1.458 \\
\hline $\mathrm{T}_{4}:$ Bavistin @ 0.5\% & 44.20 & 37.53 & 32.01 & 27.72 & 23.80 & 21.03 & 31.05 & 0.287 & 0.880 & 1.566 & 1.952 & 1.938 & 1.916 & 1.423 \\
\hline $\mathrm{T}_{5}$ :Bavistin@ $0.5 \%+\mathrm{CaCl}_{2} @ 2.0 \%$ & 45.15 & 39.90 & 33.33 & 30.15 & 25.67 & 22.14 & 32.72 & 0.289 & 0.913 & 1.629 & 1.974 & 1.955 & 1.926 & 1.447 \\
\hline $\mathrm{T}_{6}$ :Indoneem @ 500 ppm & 43.09 & 37.29 & 30.23 & 26.17 & 21.32 & 19.00 & 29.52 & 0.288 & 0.868 & 1.531 & 1.935 & 1.914 & 1.891 & 1.404 \\
\hline $\mathrm{T}_{7}$ :Indoneem @ 500 ppm + $\mathrm{CaCl}_{2} @ \mathbf{2 . 0 \%}$ & 43.62 & 38.09 & 30.99 & 26.73 & 23.31 & 20.52 & 30.54 & 0.283 & 0.907 & 1.546 & 1.943 & 1.923 & 1.901 & 1.417 \\
\hline $\mathrm{T}_{8}:$ Control & 43.54 & 34.78 & 29.18 & 24.51 & 19.66 & 17.87 & 28.26 & 0.281 & 1.147 & 1.815 & 1.789 & 1.760 & 1.727 & 1.420 \\
\hline Mean & 44.15 & 38.20 & 31.75 & 27.52 & 23.29 & 20.68 & 30.93 & 0.289 & 0.924 & 1.609 & 1.935 & 1.915 & 1.890 & 1.427 \\
\hline \multirow[t]{2}{*}{ CD at $5 \%(\mathrm{~T} \times \mathrm{D})$} & \multicolumn{6}{|c|}{0.559} & & \multicolumn{6}{|c|}{0.004} & \\
\hline & \multicolumn{14}{|c|}{ Post-harvest } \\
\hline $\mathrm{T}_{1}: \mathrm{CaCl}_{2} @ 6 \%$ & 44.52 & 39.25 & 34.77 & 25.88 & 22.05 & 19.46 & 30.99 & 0.252 & 0.874 & 1.401 & 1.910 & 1.891 & 1.872 & 1.366 \\
\hline $\mathrm{T}_{2}: \mathrm{GA}_{3} @$ 400ppm & 45.76 & 41.22 & 36.32 & 27.62 & 22.09 & 21.32 & 32.39 & 0.260 & 0.852 & 1.378 & 1.958 & 1.942 & 1.926 & 1.386 \\
\hline $\mathrm{T}_{3}: \mathrm{GA}_{3} @ 400 \mathrm{ppm}+\mathrm{CaCl}_{2} @ 6.0 \%$ & 46.47 & 41.90 & 36.50 & 28.02 & 23.71 & 21.60 & 33.03 & 0.260 & 0.846 & 1.370 & 1.971 & 1.956 & 1.941 & 1.391 \\
\hline $\mathrm{T}_{4}:$ Bavistin @ 0.1\% & 44.86 & 39.52 & 34.55 & 26.04 & 22.14 & 19.92 & 31.17 & 0.257 & 0.868 & 1.390 & 1.928 & 1.907 & 1.893 & 1.374 \\
\hline $\mathrm{T}_{5}$ :Bavistin@ 0.1\% + $\mathrm{CaCl}_{2} @ 6.0 \%$ & 45.26 & 39.94 & 35.17 & 26.53 & 22.28 & 20.20 & 31.56 & 0.255 & 0.863 & 1.368 & 1.943 & 1.925 & 1.908 & 1.377 \\
\hline $\mathrm{T}_{6}$ :Indoneem @ 500 ppm & 44.10 & 38.44 & 33.44 & 24.78 & 21.20 & 18.71 & 30.11 & 0.252 & 0.886 & 1.420 & 1.890 & 1.870 & 1.849 & 1.361 \\
\hline $\mathrm{T}_{7}$ :Indoneem @ 500 ppm + $\mathrm{CaCl}_{2} @ 6.0 \%$ & 44.63 & 39.16 & 34.08 & 25.28 & 21.26 & 19.42 & 30.64 & 0.253 & 0.879 & 1.413 & 1.898 & 1.877 & 1.858 & 1.363 \\
\hline $\mathrm{T}_{8}$ :hot water & 44.04 & 38.39 & 33.25 & 23.16 & 20.48 & 19.02 & 29.72 & 0.251 & 0.908 & 1.440 & 1.862 & 1.840 & 1.818 & 1.353 \\
\hline $\mathrm{T}_{9}$ :hot water + $\mathrm{CaCl}_{2} @ 6.0 \%$ & 43.96 & 38.29 & 33.63 & 24.68 & 20.61 & 18.51 & 29.94 & 0.251 & 0.899 & 1.430 & 1.878 & 1.857 & 1.836 & 1.358 \\
\hline $\mathrm{T}_{10}:$ Control & 44.20 & 36.33 & 30.36 & 23.90 & 20.46 & 17.82 & 28.85 & 0.254 & 1.132 & 1.764 & 1.740 & 1.716 & 1.692 & 1.383 \\
\hline Mean & 44.78 & 39.24 & 34.21 & 25.59 & 21.63 & 19.60 & 30.84 & 0.254 & 0.901 & 1.437 & 1.898 & 1.878 & 1.859 & 1.371 \\
\hline CD at $5 \%(\mathrm{~T} \times \mathrm{D})$ & \multicolumn{7}{|c|}{0.363} & \multicolumn{6}{|c|}{0.010} & \\
\hline
\end{tabular}


Table.3 Cost economics of different pre and post-harvest treatments on mango fruits cv. Dashehari (Pooled)

\begin{tabular}{|c|c|c|c|c|c|c|c|}
\hline Treatments (T) & $\begin{array}{c}\text { Fruit } \\
\text { spoilage } \\
\text { on } 12^{\text {th }} \\
\text { day (per } \\
\text { cent) }\end{array}$ & $\begin{array}{l}\text { Good } \\
\text { quality } \\
\text { mango } \\
\text { (per } \\
\text { cent) }\end{array}$ & $\begin{array}{l}\text { Sale rate } \\
\text { of } 100 \\
\text { mangos } \\
\text { (Rs.) }\end{array}$ & $\begin{array}{l}\text { Sale price } \\
\text { of good } \\
\text { quality } \\
\text { fruits (Rs.) }\end{array}$ & $\begin{array}{l}\text { Total cost of } \\
\text { treatments } \\
\text { (Rs.) }\end{array}$ & $\begin{array}{c}\text { Net } \\
\text { Profit } \\
\text { (Rs.) }\end{array}$ & $\begin{array}{l}\text { Profit } \\
\text { over } \\
\text { control } \\
\text { (Rs.) }\end{array}$ \\
\hline & & & \multicolumn{5}{|c|}{ Pre harvest } \\
\hline $\mathrm{T}_{1}: \mathrm{CaCl}_{2} @ 2 \%$ & 30.52 & 69.48 & 600 & 416.88 & 36.49 & 380.39 & 147.15 \\
\hline $\mathrm{T}_{2}: \mathrm{GA}_{3} @ 15 p p m$ & 27.85 & 72.85 & 550 & 400.67 & 35.29 & 365.38 & 132.14 \\
\hline $\mathrm{T}_{3}: \mathrm{GA}_{3} @ 15 \mathrm{ppm}+\mathrm{CaCl}_{2} @ 2.0 \%$ & 25.44 & 74.56 & 550 & 410.08 & 38.45 & 371.63 & 138.39 \\
\hline $\mathrm{T}_{4}$ :Bavistin @ $0.5 \%$ & 18.29 & 81.29 & 600 & 487.74 & 33.48 & 454.26 & 221.02 \\
\hline $\mathrm{T}_{5}$ :Bavistin@0.5\% + $\mathrm{CaCl}_{2} @ 2.0 \%$ & 19.14 & 80.86 & 600 & 485.16 & 36.64 & 448.52 & 215.28 \\
\hline $\mathrm{T}_{6}:$ Indoneem @ 500 ppm & 33.43 & 66.57 & 500 & 332.85 & 43.33 & 289.52 & 56.28 \\
\hline $\mathrm{T}_{7}$ :Indoneem @ 500 ppm + $\mathrm{CaCl}_{2} @ 2.0 \%$ & 34.11 & 65.89 & 500 & 329.45 & 49.94 & 279.51 & 46.27 \\
\hline \multirow[t]{2}{*}{$\mathrm{T}_{8}:$ Control } & 41.69 & 58.31 & 400 & 233.24 & 0.00 & 233.24 & 0.00 \\
\hline & & & \multicolumn{5}{|c|}{ Post-harvest } \\
\hline $\mathrm{T}_{1}: \mathrm{CaCl}_{2} @ 6 \%$ & 30.12 & 69.88 & 600 & 419.28 & 42.81 & 376.47 & 177.47 \\
\hline $\mathrm{T}_{2}: \mathrm{GA}_{3} @ 400 \mathrm{ppm}$ & 29.86 & 70.14 & 600 & 420.84 & 85.73 & 335.11 & 136.11 \\
\hline $\mathrm{T}_{3}: \mathrm{GA}_{3} @ 400$ ppm+CaCl $\mathrm{Cl}_{2} @ 6.0 \%$ & 28.50 & 71.50 & 600 & 429.00 & 95.21 & 333.79 & 134.79 \\
\hline $\mathrm{T}_{4}$ :Bavistin @ 0.1\% & 18.33 & 81.67 & 650 & 530.85 & 33.36 & 497.49 & 298.49 \\
\hline $\mathrm{T}_{5}$ :Bavistin@ $0.1 \%+\mathrm{CaCl}_{2} @ 6.0 \%$ & 18.93 & 81.07 & 650 & 526.95 & 42.84 & 484.11 & 285.11 \\
\hline $\mathrm{T}_{6}$ :Indoneem @ 500 ppm & 30.78 & 69.22 & 500 & 346.10 & 43.33 & 302.77 & 103.77 \\
\hline $\mathrm{T}_{7}$ :Indoneem @ 500 ppm + $\mathrm{CaCl}_{2} @ 6.0 \%$ & 32.01 & 67.99 & 500 & 339.95 & 52.81 & 287.14 & 88.14 \\
\hline $\mathrm{T}_{8}$ :hot water & 17.09 & 82.91 & 700 & 580.37 & 33.33 & 547.04 & 348.04 \\
\hline $\mathrm{T}_{9}$ :hot water + $\mathrm{CaCl}_{2} @ 6.0 \%$ & 14.80 & 85.20 & 700 & 596.40 & 42.81 & 553.59 & 354.59 \\
\hline $\mathrm{T}_{10}:$ Control & 50.25 & 49.75 & 400 & 199.00 & 0.00 & 199.00 & 0.00 \\
\hline
\end{tabular}


The decrease in ascorbic acid is due to oxidative destruction of ascorbic acid in the presence of molecular oxygen by ascorbic acid oxidase enzyme (Kirmani et al., 2013).

$\beta$-carotene content of the fruits treated with chemicals increased up to $9^{\text {th }}$ day and after attaining the respective peaks, it declined with advancement of storage period till $14^{\text {th }}$ day of storage. The maximum $\beta$-carotene content was obtained by pre harvest spray of Gibberellic acid@ 15 ppm + Calcium chloride @ 2.0\%

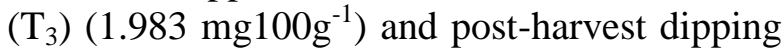
with $\mathrm{GA}_{3} @ 400$ ppm + $\mathrm{CaCl}_{2} @ 6.0 \%\left(\mathrm{~T}_{3}\right)$ $\left(1.971 \mathrm{mg} 100 \mathrm{~g}^{-1}\right)$ on $9^{\text {th }}$ day of storage. The decline in $\beta$-carotene might be due to their utilization in evapo-transpiration and other biochemical activities (Bhatt et al., 2012; Yadav et al., 2010).

Estimation of profit for 100 fruits over control (Table 3) indicated that maximum net profit (Rs.221.02 and Rs.354.59, respectively) was obtained from fruits of pre-harvest treatment Bavistin @ $0.5 \% \quad\left(\mathrm{~T}_{4}\right)$ and post-harvest treatment Hot water dipping + Calcium chloride @ $6.0 \%\left(\mathrm{~T}_{9}\right)$. Net profit of control fruits was taken as Rs. 0.00 for comparison with all treated fruits.

Thus, it may be concluded that the pre harvest spray with Gibberellic acid @ 15 ppm + Calcium chloride@2.0\% and post-harvest dipping with $\mathrm{GA}_{3} @ 400$ ppm $+\mathrm{CaCl}_{2} @ 6.0 \%$ and Hot water + Calcium chloride @ 6.0\% are the most effective treatments for increased net profit, storage life as well as maintained fruit quality of mango cv. Dashehari.

\section{References}

A.O.A.C. 1990. Official Methods of Analysis. Association of Official Analytical Chemists, Washington, D.C.
Bhatt, A., N.K Mishra, D.S. Mishra and C.P. Singh 2012. Foliar application of potassium, calcium, zinc and boron enhanced yield, quality and shelf life of mango. Hort-Flora Research Spectrum, 1(4): 300-305.

Choudhury, S., D.P. Ray, B.K. Das and G.S. Sahu, 2003. Effect of pre and post-harvest chemical treatments on ripening, quality and storage life of sapota (Manilkara achras (Mill.) Forberg) cv. Pala. Orissa Journal of Horticulture, 31(2): 54-57.

Cisneros-Zevallos, L. 2003. The use of controlled postharvest abiotic stresses as a tool for enhancing the nutraceutical content and adding-value of fresh fruits and vegetables. Journal of Food Science, 68: 1560-1565.

Kirmani, S.N., G.M. Wani, M.S. Wani, M.Y. Ghani, M. Abid, S. Muzamil, H. Raja and A.R. Malik, 2013. Effect of preharvest application of Calcium chloride $\left(\mathrm{CaCl}_{2}\right)$, Gibberellic acid $\left(\mathrm{GA}_{3}\right)$ and Napthelenic acetic acid (NAA) on storage of Plum (Prunus salicina L.) cv. Santa Rosa under ambient storage conditions. African Journal of Agricultural Research, 8(9): 812-818.

Mahajan, B.V.C., A.S. Dhatt, and W.S. Dhilon, 2004. Effect of pre-storage treatments on quality and storage life of Asian pear. Indian Journal of Horticulture, 61(4): 342344.

Wahdan, M.T., S.E. Habib, M.A. Bassal, and E.M. Qaoud, 2011. Effect of Calcium chloride and Gibberellic acid on storability of "Succary Abiad" mango fruits under cold storage. Journal of American Science, 7(5): 493-501.

Yadav, M., N. Kumar, D.B. Singh, and G.K. Singh, 2010. Effect of post-harvest treatments on shelf life and quality of Kinnow mandarin. Indian Journal of Horticulture, 67(2): 243-248.

\section{How to cite this article:}

Pant, Pooja and Singh, C.P. 2018. Response of Pre and Post-Harvest Chemical Applications on Storage Life, Quality and Cost Economics of Mango cv. Dashehari in Tarai Region of Uttarakhand, India. Int.J.Curr.Microbiol.App.Sci. 7(11): 2755-2760. doi: https://doi.org/10.20546/ijcmas.2018.711.316 